\title{
UNA COMBINACIÓN NUEVA EN PHEMERANTHUS (PORTULACACEAE)
}

\author{
Gilberto Ocampo Acosta ${ }^{1}$ \\ Instituto de Ecología, A.C. \\ Centro Regional del Bajío \\ Apartado Postal 386 \\ 61600 Pátzcuaro, Michoacán \\ ocampo@inecolbajio.edu.mx
}

\section{RESUMEN}

Se propone como combinación nueva a Phemeranthus oligospermus (Brandegee) G. Ocampo, con el propósito de validar el nombre para la publicación del fascículo correspondiente a la familia Portulacaceae para la Flora del Valle de Tehuacán-Cuicatlán, México. Adicionalmente, se hace un comentario acerca de la información de las etiquetas que poseen los ejemplares isotipo de la especie. Cuicatlán.

Palabras clave: México, Phemeranthus, Portulacaceae, Talinum, Valle de Tehuacán-

\section{ABSTRACT}

Phemeranthus oligospermus (Brandegee) G. Ocampo is proposed here as a new combination in order to validate the name for publication of the treatment of Portulacaceae for the Flora of Valley of Tehuacán-Cuicatlán, Mexico. In addition, a comment concerning the information given on the labels of isotype specimens of this species is included.

Key words: Mexico, Phemeranthus, Portulacaceae, Talinum, Valley of Tehuacán-Cuicatlán.

Recientemente, algunos estudios moleculares en el género Talinum Adanson (Hershkovitz y Zimmer, 1997; Applequist y Wallace, 2001) sugieren la segregación de la sección Phemeranthus (Raf.) DC. a nivel genérico. Estos datos concuerdan con evidencias morfológicas (Carolin, 1993), pues algunos caracteres de la hoja, del polen y del fruto, entre otros, pueden utilizarse para separar las dos entidades. Tal esquema ha sido, en general, bien aceptado, por lo que Phemeranthus Raf. será reconocido como componente de la familia Portulacaceae en las contribuciones que se encuentran en preparación para la Flora of North America (Kiger, 2001) y para la Flora del Bajío y de Regiones Adyacentes (Ocampo, 2002).

1 Trabajo realizado con apoyo económico del Instituto de Ecología, A.C. (cuenta 902-07), del Consejo Nacional de Ciencia y Tecnología y de la Comisión Nacional para el Conocimiento y Uso de la Biodiversidad. 
El género Phemeranthus Raf. (Specchio Sci. 1(3): 86. 1814) se caracteriza por poseer hojas rollizas a subrollizas, generalmente angostas, por el polen polirrugado, por la cápsula con valvas que pronto se desprenden, así como por el epicarpo someramente diferenciado que no se separa del endocarpo y por las semillas con testa lisa o con surcos concéntricos, no tuberculada en toda su superficie, que en la mayoría de las especies están cubiertas por una película translúcida. Talinum posee hojas aplanadas, anchas, polen poliforado, cápsulas con valvas que caen tardíamente, con algunas valvas o parte de las mismas persistentes, el epicarpo frecuentemente se encuentra diferenciado y por lo común se separa del endocarpo fibroso, las semillas tienen diminutos tubérculos en toda su superficie y, en la mayoría de las especies, tienen un pequeño arilo cerca de la base.

Phemeranthus es un género americano, con alrededor de 27 especies distribuidas en Norteamérica, con la única excepción de Talinum punae (Fries) Carolin, taxon de distribución sudamericana. Algunas de estas especies aún se mantienen en el género Talinum, en espera de su revisión y posible transferencia y, de esta forma, hacer más aceptable la delimitación de ambos géneros. En tal situación se encuentra Talinum oligospermum Brandegee, entidad que requiere de tal cambio, para validar el nombre que será reconocido en el fascículo correspondiente a la familia Portulacaceae de la Flora del Valle de Tehuacán-Cuicatlán, México.

Phemeranthus oligospermus (Brandegee) G. Ocampo, comb. nov. Basónimo: Talinum oligospermum Brandegee, Zoe 5: 245. 1908. Tipo: México. Puebla: Collected on Cerro de la Yerba, growing in dry, rocky soil, July 1907, C. A. Purpus 2513 (holotipo: UC (imagen!?); isotipos: MO (imagen! ${ }^{3}$ ), NY (imagen! $\left.{ }^{4}\right)$, US (microficha Chadwyck-Healy No. 0260/D03!)).

Se trata de una especie endémica, conocida únicamente de localidades ubicadas en los límites entre los estados de Oaxaca y Puebla, en sitios con vegetación de bosque de encino y de matorral espinoso, entre 2000 y $2600 \mathrm{~m}$ de altitud. Este taxon se registra como abundante localmente; no obstante las colecciones que lo representan son muy escasas, por lo que puede considerarse como una especie rara.

Brandegee, en la parte introductoria de su artículo concerniente a las especies nuevas de plantas mexicanas (Zoe 5: 244. 1908), señala que hace las descripciones con base en colectas efectuadas por Purpus entre los años 1906 y 1907 en los estados de Puebla y Oaxaca. En la descripción de Talinum oligospermum únicamente proporciona la localidad de colecta, mas no el año en que ésta se efectuó (Zoe 5: 246. 1908) y tal omisión ha dado cabida a una confusión en cuanto a la fecha de la misma. Rose y Standley (1911), en su trabajo sobre el género Talinum en México, refieren la fecha de colecta como "June, 1908" (p. 285), lo mismo que indican los bancos de datos de los herbarios $\mathrm{MO}$, NY y US ${ }^{5}$, que conservan los ejemplares isotipo de la especie. Al revisar las etiquetas de tales especímenes, se observa la anotación "Collected in the vicinity of

\footnotetext{
${ }^{2}$ University and Jepson Herbaria, University of California, Berkeley (http://ucjeps.herb.berkeley.edu).

3 Missouri Botanical Garden, w3TROPICOS (http://mobot.mobot.org/W3T/Search/vast.html).

${ }^{4}$ New York Botanical Garden, Vascular Plant Type Catalog (http://www.nybg.org/bsci/hcol/vasc/).

${ }_{5}$ Smithsonian Institution, Botanical Type Specimen Register (http://rathbun.si.edu/botany/types/).
} 
San Luis Tultitlanapa, Puebla, near Oaxaca; C. A. Purpus 2513; June 1908”, información que no concuerda con el sitio de colecta ni con la fecha indicada por Brandegee en la descripción original. Por otra parte, Sousa (1969) hace un recuento de los sitios explorados por Purpus y señala que la localidad "Cerro de la Yerba" fue visitada en los meses de julio y agosto de 1907 (p. 21) y finalmente, al revisar los datos de la etiqueta de colecta del ejemplar holotipo, depositado en UC, se lee lo siguiente: "Cerro de la Yerba, Puebla; C. A. Purpus 2513; July 1907; sunny, dry, rocky soil, 7-8000ft", datos que sí coinciden con lo señalado por Brandegee.

Se sabe que Purpus no efectuaba descripciones muy detalladas de sus localidades, además de que sus especímenes fueron numerados mucho tiempo después y que tal numeración no seguía el orden cronológico de colecta (Sousa, op. cit., p. 15). En tales circunstancias, cabe considerar que los ejemplares isotipo podrían no corresponder al mismo juego colectado en "Cerro de la Yerba", o bien, que para estos ejemplares las etiquetas de colecta no hayan sido elaboradas cuidadosamente, error que pudiera atribuirse al personal del herbario UC, encargado de organizar y distribuir los duplicados de Purpus, o al propio Brandegee. En consecuencia, la fecha de colecta del tipo debe citarse como efectuada en julio de 1907, tal y como se indica en el ejemplar holotipo.

\section{AGRADECIMIENTOS}

Deseo reconocer la ayuda de Rosalinda Medina en la consecución de literatura, así como a Jerzy Rzedowski la revisión crítica del manuscrito.

\section{LITERATURA CITADA}

Applequist, W. L. y R. S. Wallace. 2001. Phylogeny of the portulacaceous cohort based on ndhF sequence data. Syst. Bot. 26(2): 406-419.

Carolin, R. C. 1993. Portulacaceae. In: Kubitzki K., J. G. Rohwer y V. Bittrich (eds.). The families and genera of vascular plants. Vol. 2. Springer-Verlag. Nueva York. pp. 544-555.

Hershkovitz, M. A. y E. A. Zimmer. 1997. On the evolutionary origins of the cacti. Taxon 46(2): 217232.

Kiger, R. W. 2001. New combinations in Phemeranthus Rafinesque (Portulacaceae). Novon 11(3): 319-321.

Ocampo, G. 2002. Transferencia de tres especies mexicanas de Talinum Adans. a Phemeranthus Raf. (Portulacaceae). Acta Bot. Mex. 59: 75-80.

Rose, J. N. y P. C. Standley. 1911. The genus Talinum in Mexico. Contr. U. S. Nat. Herb. 13(8): 281288.

Sousa, S. M. 1969. Las colecciones botánicas de C. A. Purpus en México. Período 1898-1925. Univ. Calif. Publ. Bot. 51: 1-36. 